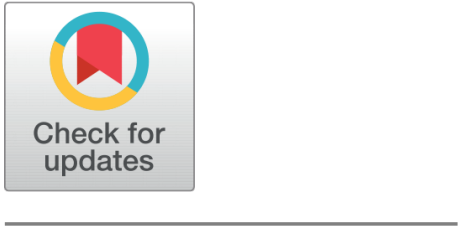

OPEN ACCESS

Received: 10.10 .2020

Accepted: 29.01.2021

Published: 22.02 .2021

Citation: Sugunakar MBS, Maruthy KN, Srinivas $\mathrm{CH}$, Johnson $\mathrm{P}$ (2021) A comparative study between single lead AD8232 heart rate monitor and standard electrocardiograh to acquire electrocardiographic data for cardiac autonomic function testing. Indian Journal of Science and Technology 14(6): 534-540. https://d oi.org/10.17485/IJST/v14i6.1822

* Corresponding author.

Tel: +919884348585

priscillajohnson@sriramachandra.edu.in

Funding: None

Competing Interests: None

Copyright: @ 2021 Sugunakar et al. This is an open access article distributed under the terms of the Creative Commons Attribution License, which permits unrestricted use, distribution, and reproduction in any medium, provided the original author and source are credited.

Published By Indian Society for Education and Environment (iSee)

ISSN

Print: 0974-6846

Electronic: 0974-5645

\section{A comparative study between single lead AD8232 heart rate monitor and standard electrocardiograh to acquire electrocardiographic data for cardiac autonomic function testing}

\author{
M B S Sugunakar ${ }^{1}$, K N Maruthy ${ }^{2}$, C H Srinivas ${ }^{3}$, Priscilla Johnson ${ }^{4 *}$ \\ 1 Tutor, Department of Physiology, G.S.L. Medical College, Rajahmundry, A P, India \\ 2 Professor and HOD, Department of Physiology, Narayana Medical College, Nellore, A P \\ 3 Professor and HOD, Department of Physiology, G.S.L.Medical College, Rajahmundry, A P \\ 4 Professor \& HOD, Department of Physiology, Sri Ramachandra Medical College \& Research \\ Institute, SRIHER (DU), Porur, Chennai, Tel.: +919884348585
}

\section{Abstract}

Objectives: The present study was to develop and design a simple and low cost ECG setup for patient care. The device is a low cost and simple approach for acquiring ECG data. The present study mainly focuses on reliability and consistency for acquiring the ECG data. It should be simple to use, portable and low cost to implement. Methods: The following study was conducted between two groups of subjects; each group contains $n=40$ subjects. First the subjects were recruited to the department of physiology autonomic function laboratory and the demographic and anthropometric data was acquired. The subjects were allowed to rest for some time. Single lead ECG was obtained using AD8232 custom designed module and was connected to a laptop as signal was captured by audacity software and compared against a medical grade ECG monitor and the results were analyzed. Findings: From our study it is clearly evident that there is a strong positive correlation of ECG data obtained between AD8232 and standard clinically approved ECG monitor $r=0.9(p \leq 0.001)$. Now it is clearly evident that we can use AD8232 module instead of standard ECG device for basic monitoring. Novelty: The new device developed successfully captured single lead ECG which is a low cost implementation around ₹2350 (table given in annexure). Capturing ECG with microphone input is quite new way of acquiring ECG signals. The new device was easy to set up, no special software is needed to acquire the signal runs on USB voltage which is $5 \mathrm{v}$. The device is simple to use, open source hardware and consistent with the recordings when compared with the standard ECG monitor. The latest device can be configured in many ways for future applications using open source sound capturing software. Audacity open source software.

Keywords: Portable ECG; single lead; USB; open source; AC; DC; AD8232; GPL 


\section{Introduction}

Cardio vascular diseases were one of the leading causes of deaths worldwide in $2011^{(1)}$. Electrocardiography has been one of the oldest and efficient techniques for detecting the electrical activities of the myocardium. ECG machines evolved long way since their inception from a single channel to 12 channels machines which are being used nowadays ${ }^{(2)}$. Electrocardiography is considered as a very valuable diagnostic tool for evaluating a number of cardio vascular diseases (CVD) ranging from simple arrhythmias to complex heart blocks ${ }^{(3)}$. After many advancements and incremental developments to the ECG devices with ultimately lead to development of portable machines from old devices which were around 500 pounds at the time of Einthoven who awarded noble prize for his invention ${ }^{(4)}$. The three-lead ECG usage expanded significantly commercialized and standardized ${ }^{(4)}$. Many improvements were made to make it more portable especially using the latest software programming languages ${ }^{(5,6)}$. There are many significant portable devices are also developed with the AD823X module using Arduino platform however they required 2 to 3 PCB boards to operate and special python program to execute signal acquiring code ${ }^{(7)}$. The current device was a low cost implementation which is an improved version with single PCB and quite portable compared to the other single lead ECG devices. The current device was able to address some of the key challenges mentioned by other devices regarding the filtration of noise and improving the signal to noise ratio by applying low pass and hi pass digital filters ${ }^{(8)}$. Our device was developed to acquire the ECG signal with low cost (anx1) without any special software can be implemented easily even in the rural Indian setup where the resources are very limited, only by using a laptop and my portable ECG device. The current device will bring ECG recording into very practical and easy to record level.

\section{Waves of ECG}

1. P-wave : caused due to atrial depolarization

2. QRS complex : atrial repolalization, ventricular depolarization

3. T-wave ventricular repolarizaton.

Based on the above data a physician can identify the abnormalities and pathologies in the cardiovascular system.

AD8232 is a low cost solution for acquiring ECG data since conventional ECG monitors are bulky and costly. Being a mobile and versatile device can be used in outpatient setups near physician for acquiring ECG data. Being an open source software, and hardware can be configured to analysis other bio-electrical potentials like EEG, EMG, etc. The device can be used to demonstrate ECG signals without wasting any paper just by using PC monitor for the students in teaching sessions.

\section{Materials and Methods}

- The current device is procured from an open source development firm sparkfun design and uses an analog device IC model no AD8232 which is widely available and commonly used home based and low cost ECG signal acquiring devices.(Figure 1). The AD8232 device connected to microphone input jack of laptop with audio processing hardware developed by Realtek having 16bit audio driver with $44 \mathrm{khz}$ sample rate and specifications were given in the spec sheet attached with this document. The device uses 3 electrodes; first electrode connected to right arm second one to left arm and third one connected to right leg which is ground electrode. All three electrodes were color coded. The electrodes were not interchangeable. A modified and $3.5 \mathrm{~mm}$ male jack adapter designed by me is connected to output pins of ECG module and other side is analog input jack with microphone adapter which was specifically developed for the purpose of connecting the controller to the laptop. No intermediate PCB was needed in my implementation. The sparkfun module is directly connected to laptop microphone input $3.5 \mathrm{~mm}$ jack. USB is only used for power to drive the circuit. Microphone boost $+10 \mathrm{db}$ commonly used to improve $\mathrm{S} / \mathrm{N}$ ratio. Avoided the AC power adapter to the laptop to reduce further noise.

- For standard ECG monitors, all the parameters like PR interval, QRS interval, and Heart rate were evaluated using normal counting method of squares on ECG paper. The signal is captured using audacity open source software. Then the $40 \mathrm{~Hz}+10 \mathrm{~Hz}$ low pass filter is applied. With beat finder the R-R interval is labeled, the file is exported to MS Excel and R-R interval is calculated then the file is imported to kubios HRV software to get a detailed report. Time stamps of different waves were displayed on the tool bar of the audacity software and were recorded. Audacity, an open source sound recording and capturing software, was installed on PC running windows 10 to capture and analyze the ECG signal.

- Total 80 normal participants without any cardiovascular conditions aged between 21 to 55 years were selected for the study and divided into two groups $n=40$.

ECG was recorded using Maestros Magic-R model No MRX -12 ECG (Maestros - Cardiology Equipment) acquired from the cardiology department in G.S.L. General hospital an officially certified monitor which was having paper output 
(Figure 2)

ECG was obtained again for the same subject this time using AD8232 portable setup with laptop and saved in the audacity software for the further analysis (fig .3)

\section{Results and Discussion}

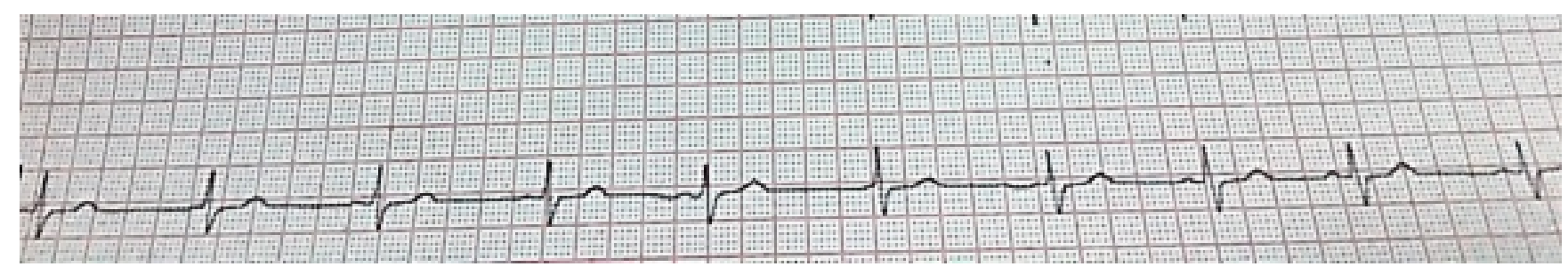

Fig 1. ECG recordings using standard ECG monitor

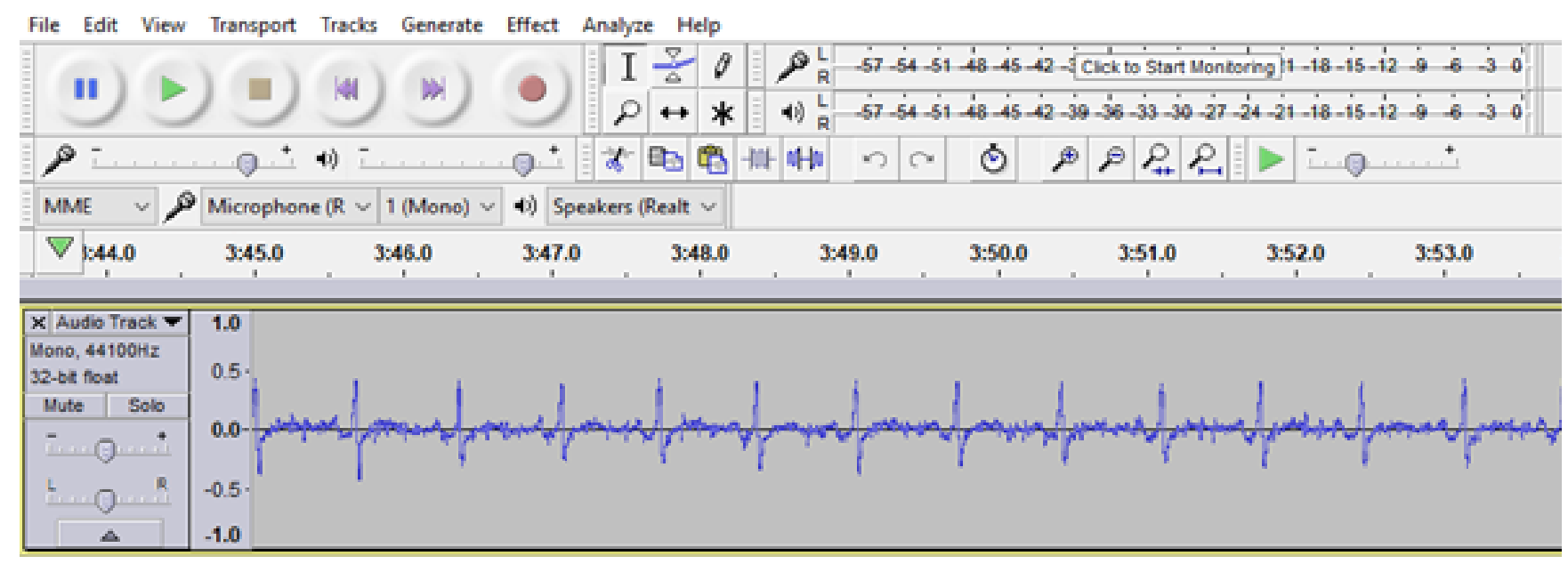

Fig 2. ECG recording usingAD8232 setup

\section{Analysis and comparison of acquired data}

According to the above data, AD8232 can be used as an alternative to standard ECG monitor for basic monitoring and analysis of ECG which is statistically significant with $(\mathrm{p} \leq 0.001)$. The advantages of present device is we can use a simple ECG module with a laptop most of the ECG modules were either costly, requires proprietary hardware requires a programming a hardware like raspberry pi with python or other embedded systems ${ }^{(9)}$. This hardware being open source easy to implement and runs on open source software without any knowledge in the software programming. Physician often don't have a thorough idea regarding the computer programming languages. Being a simple accessory to a laptop with very portable equipment a physician can easily acquire the ECG data without any costly setup. The working prototype was designed by me and tested for consistency and the results were promising the photographs of the prototype, circuit diagrams were enclosed with the article. The statistical analysis was performed using MS excel and online statistical software analysis platform (sociostatiscs, graphpad prizm) means of all values with their SD were calculated. The Table 1 shows mean values of ECG signal data of two groups $\mathrm{n}=40$ and their correlation coefficient " $r$ ". 
Table 1. ECG signal data of two groups $n=40$

\begin{tabular}{llll}
\hline \multirow{2}{*}{ Variables in their mean values } & \multicolumn{2}{c}{ Supine position } & Correlation coefficient \\
\cline { 2 - 4 } & AD8232 & ECG monitor & $0.9,(\mathrm{p} \leq 0.001)$ \\
\hline Mean HR $(\mathrm{bpm})$ & $83 \pm 14.1$ & $84 \pm 13.9$ & $0.9,(\mathrm{p} \leq 0.001)$ \\
PR interval (sec) & $0.16 \pm 0.01$ & $0.17 \pm 0.01$ & $0.7,(\mathrm{p} \leq 0.001)$ \\
QRS interval(sec) & $0.13 \pm 0.01$ & $0.14 \pm 0.01$ & $0.9,(\mathrm{p} \leq 0.001)$ \\
QT interval (sec) & $0.36 \pm 0.07$ & $0.36 \pm 0.07$ & $0.9,(\mathrm{p} \leq 0.001)$ \\
RR interval $(\mathrm{sec})$ & $0.72 \pm 0.15$ & $0.73 \pm 0.15$ & \\
\hline
\end{tabular}

\section{Discussion}

From the above study it is clearly evident that there is a strong positive correlation of ECG data obtained between AD 8232 and standard clinically approved ECG monitor $\mathrm{r}=0.9(\mathrm{p} \leq 0.001)$. Hence, we can use AD8232 module instead of standard ECG device for basic monitoring; however, there are certain pros and cons in usage of both devices which are discussed below.

Pros: AD8232 versatile device, easily available for purchase through online. The device was tested with clamp electrodes without applying any gel so discomfort and irritation caused due to usage gel can be avoided. The device run on $3 \mathrm{~V}$ to $5 \mathrm{~V}$ DC which is the most common USB voltage that can be obtained by connecting to a USB port no need to find an AC power socket. If we change the placement of the chest lead we can mimic the function of the functions of the multichannel ECG machine because most of the cardiovascular abnormalities were consistent along each cardiac cycle it is convenient to record multiple channels ECG if resources are available but not absolutely necessary for diagnosing a cardiovascular condition. The detection QRS complex has a great significance in the development of automated intelligent ECG signal detection process and may algorithms were also developed from past 30 years by the researches on the basis of digital filters ${ }^{(10)}$.

Cons: only basic monitoring can be achieved. For analysis of axis deviation, a real time 12 channel ECG is required. Most recent ECG monitors have advanced signal processors and patent information storage capabilities. Scope of further development: the more compact sound recording software can be developed which can run on mobile devices since audacity software is open source and comes under the terms of GPL.

\section{Conclusion}

The device may not be a groundbreaking implementation for recording of the ECG however; it can solve some practical and technical problems faced using conventional ECG machines. Here are the some key features of the device.

1. Portability: the device is quite portable and easy to connect and carry along with the laptop as most of the physicians were having personal laptop with them.

2. Ease of use: The simple step by step guidelines about using the device was given; a normal person can also connect and acquire the ECG recording in home.

3. Affordability: Very cost effective as the whole device can cost less than ₹3000. There is no need to waste paper and ink since data is stored in the hard disk of the computer.

4. Scope of improvement: Design was not patented and all the software and hardware were open source so anyone can modify and improvise the device. 


\section{Supplementary Materials}
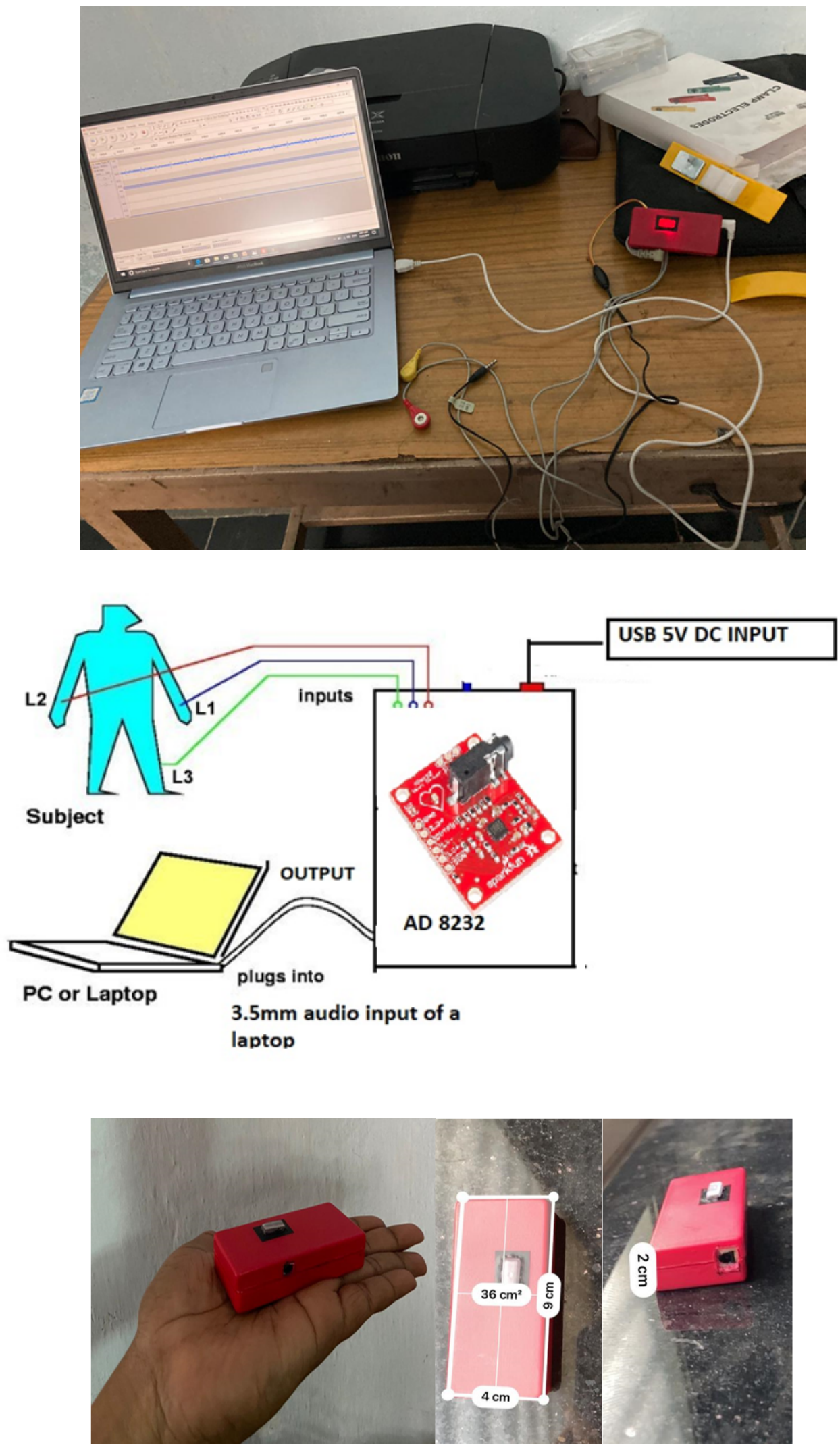


\section{AD 8232 pin configuration}

\section{FEATURES}

Fully integrated single-lead ECG front end

Low supply current: $170 \mu \mathrm{A}$ (typical)

Common-mode rejection ratio: $80 \mathrm{~dB}$ (dc to $60 \mathrm{~Hz}$ )

Two or three electrode configurations

High signal gain $(G=100)$ with dc blocking capabilities

2-pole adjustable high-pass filter

Accepts up to $\pm 300 \mathrm{mV}$ of half cell potential

Fast restore feature improves filter settling

Uncommitted op amp

3-pole adjustable low-pass filter with adjustable gain

Leads off detection: ac or dc options

Integrated right leg drive (RLD) amplifier

Single-supply operation: $2.0 \mathrm{~V}$ to $3.5 \mathrm{~V}$

Integrated reference buffer generates virtual ground

Rail-to-rail output

Internal RFI filter

$8 \mathrm{kV}$ HBM ESD rating

Shutdown pin

20-lead, $4 \mathrm{~mm} \times 4 \mathrm{~mm}$ LFCSP and LFCSP_SS package

Qualified for automotive applications
FUNCTIONAL BLOCK DIAGRAM

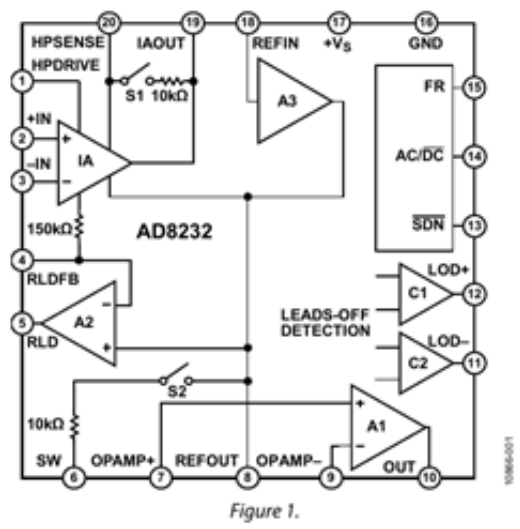

\section{Connection layout of ECG module}$$
4
$$
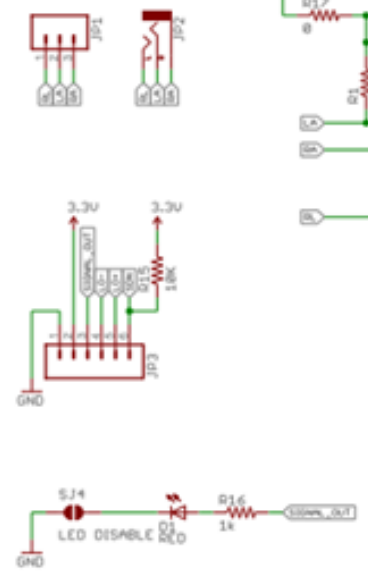

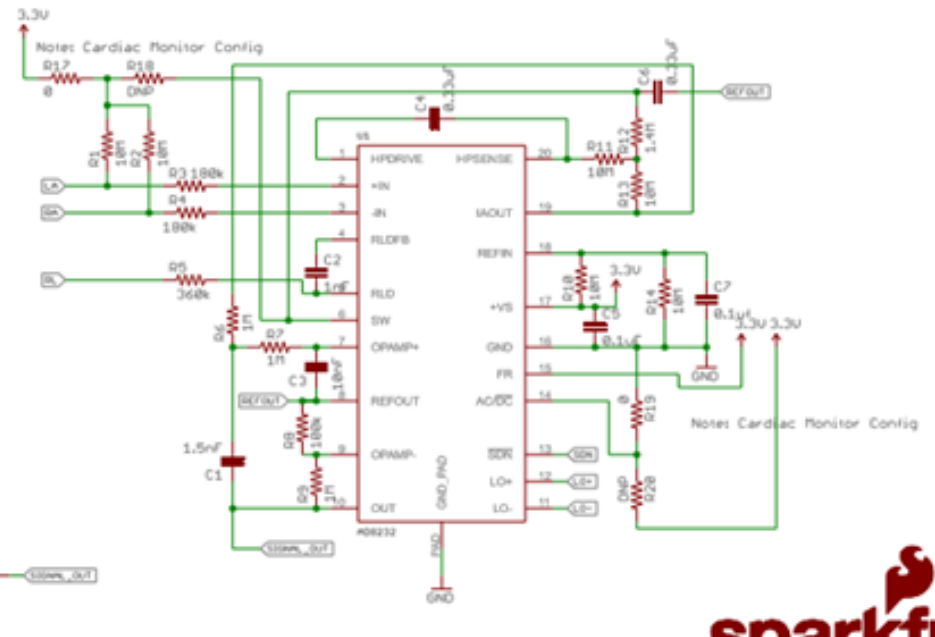

sparkfun

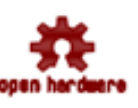

Aeleased under the Creative Commons $\circ \circ$ hupa//ereativecommons.org/licenses/oy-sa/3.a 000

TITLE: A08232_Heart_Rate_Monitor_v10

\begin{tabular}{|l|l|}
\hline Design byzasey Kuhns & REU: \\
vio
\end{tabular}

\begin{tabular}{|l|l|l|}
\hline Date: 7/15/2014 11:07:00 AM & Sheet: $1 / 1$
\end{tabular} 


\section{HRV analysis of the acquired data using KUBIOS software}

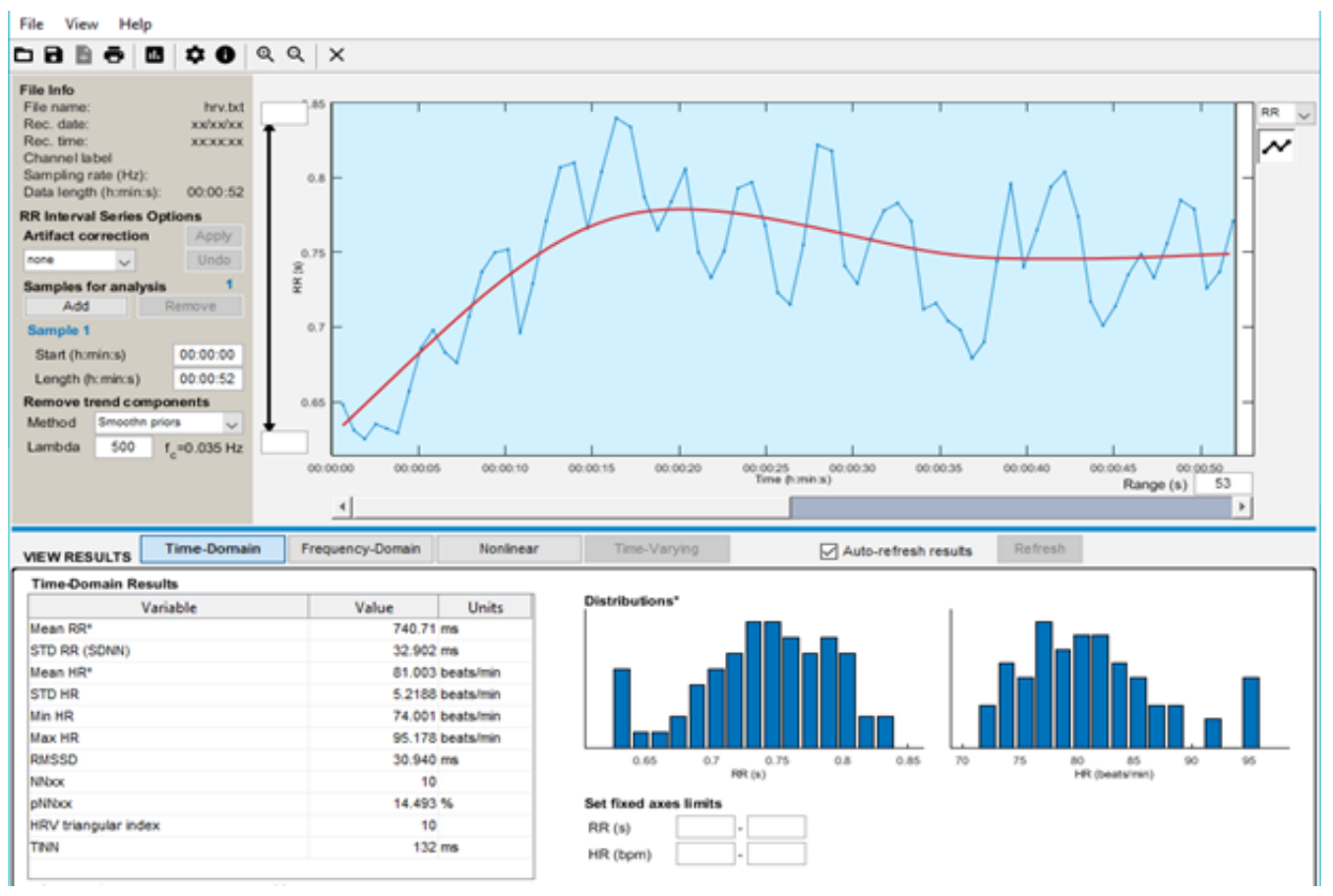

\section{Cost of implementation AD 8232}

\begin{tabular}{ll}
\hline COMPONENT & COST IN RUPEES \\
\hline AD8232 module /BOX & 1200 \\
Clamp electrodes & 450 \\
Input microphone adapter custom made & 150 \\
Sticker electrodes for additional use & 250 \\
Wires and pins & 200 \\
Storage box & 100 \\
Total cost & 2350 \\
\hline
\end{tabular}

\section{References}

1) World Health Organization. Global Atlas on Cardiovascular Disease Prevention And Control. Policies, Strategies and Interventions. Iraq. 2011. Available from: www.who.int.

2) Dhakne GM, Bhandaru SH, Mitkar AH. Design of Cost and Power Efficient Wireless Remote Cardiac Patient Monitoring System. .

3) American TN. Task Force of the European Society of Cardiology and The North American Society of Pacing and Electrophysiology. Eur Heart J [Internet]. 1996;17:354-381. Available from: http://www.mendeley.com/research/guidelines-heart-rate-variability-2/.

4) AlGhatrif M, Lindsay J. A brief review: history to understand fundamentals of electrocardiography. Journal of Community Hospital Internal Medicine Perspectives. 2012;2(1). Available from: https://dx.doi.org/10.3402/jchimp.v2i1.14383.

5) Xie C. Leveraging Data Science for Global Health [Internet]. Springer International Publishing. . Available from: http://dx.doi.org/10.1007/978-3-03047994-7_17.

6) Maheshkumar K, Dilara K, Maruthy KN, Sundareswaren L. Validation of PC-based sound card with Biopac for digitalization of ECG recording in shortterm HRV analysis. North American Journal of Medical Sciences. 2016;8(7). Available from: https://dx.doi.org/10.4103/1947-2714.187150.

7) Bravo-Zanoguera M. Development, Validation. sensors .. Available from: https://doi.org/10.3390/s20205962.

8) Serhani MA, Kassabi HTE, Ismail H, Navaz AN. ECG Monitoring Systems: Review, Architecture, Processes, and Key Challenges. Sensors. 2020;20. Available from: https://dx.doi.org/10.3390/s20061796.

9) Yin M, Tang R, Liu M, Han K, Lv X, Huang M, et al. Influence of Optimization Design Based on Artificial Intelligence and Internet of Things on the Electrocardiogram Monitoring System. Journal of Healthcare Engineering. 2020;2020:1-8. Available from: https://dx.doi.org/10.1155/2020/8840910.

10) Zhang Z, Li Z, Li Z. An Improved Real-Time R-Wave Detection Efficient Algorithm in Exercise ECG Signal Analysis. J Healthc Eng. Available from: https://www.hindawi.com/journals/jhe/2020/8868685/. 